\title{
Prenatal Diagnosis of Fetal Arrhythmias and Assessment of Autonomic Nervous System Activity by Fetal Magnetocardiography
}

\author{
Akimune Fukushima ${ }^{1, *}$, Atsushi Matsumoto ${ }^{2}$, Miyuki Terata ${ }^{3}$, Tomonobu Kanasugi ${ }^{3}$ and Kenji Nakai ${ }^{4}$ \\ ${ }^{I}$ Department of Clinical Genetics, School of Medicine, Iwate Medical University, Morioka, Iwate, Japan \\ ${ }^{2}$ Department of Pediatrics, School of Medicine, Iwate Medical University, Morioka, Iwate, Japan \\ ${ }^{3}$ Department of Obstetrics and Gynecology, School of Medicine, Iwate Medical University, Morioka, Iwate, Japan \\ ${ }^{4}$ Department of Dentistry and Internal Medicine, School of Dentistry, Iwate Medical University, 19-1 Uchimaru, \\ Morioka 020-8505 Japan
}

\begin{abstract}
Magnetocardiography (MCG) is a technique that enables the noncontact, noninvasive measurement of electrocardiographic phenomena, and fetal magnetocardiography (FMCG) is extremely useful for the analysis of fetal electrocardiographic phenomena. We have used FMCG for the diagnosis and treatment of a range of fetal arrhythmias, and to perform comparative studies of autonomic nervous system activity in fetuses with normal growth and those with fetal growth restriction by analyzing fetal heart rate variability. In terms of fetal arrhythmias, fatal long QT syndrome was accurately diagnosed prenatally using FMCG, and pregnancy was maintained until delivery at 37 weeks gestation. With regard to fetal autonomic nervous system activity, there was a pronounced increase in sympathetic nervous activity among fetuses with normal growth in the second half of pregnancy.
\end{abstract}

Keywords: Fetus, arrhythmias, autonomic nervous system activity, fetal growth restriction, magnetocardiography, prenatal diagnosis, long QT syndrome.

\section{INTRODUCTION}

Appropriate assessment of fetal information is extremely important in perinatal management and treatment. Recent advances in medical electronics in fields such as ultrasonography and cardiotocography have enabled the acquisition of a wide range of fetal information, and dramatic strides have also been made in the accuracy of prenatal diagnosis. Nevertheless, it remains difficult to obtain appropriate information by conventional methods in areas such as fetal electrocardiographic phenomena and central nervous system activity.

Magnetocardiography (MCG) is a technique that utilizes a superconducting quantum interference device (SQUID) for three-dimensional extracorporeal analysis of the minute magnetic fields generated by electrocardiographic phenomena in the heart. MCG has the following features not possessed by electrocardiography (ECG): (1) it enables noncontact measurement; (2) it offers outstanding temporospatial resolution; (3) unlike ECG, which measures scalar quantities, it measures vector quantities, enabling assessment in three dimensions (X, Y, Z); (4) because magnetic permeability is constant, unaffected by fat or neighboring organs, theretus, including a SQUID fluxmeter utilizing liquid helium for measurement and a magnetically shielded room.

As MCG enables noncontact, noninvasive measurement, fetal MCG (FMCG) is regarded as exceptionally useful for the analysis of fetal electrocardiographic phenomena. In re-

*Address correspondence to this author at the 19-1 Uchimaru, Morioka, Iwate 020-8505 Japan; Tel: +81-19-651-5111; Fax: +81-19-651-5111;

E-mail: akimunef@iwate-med.ac.jp
Table 1. Fetal arrhythmia Cases Analyzed by MCG

\begin{tabular}{|l|c|}
\hline \multicolumn{1}{|c|}{ Total } & 10 cases \\
\hline \hline Premature ventricular contraction (PVC) & 4 \\
\hline Premature atrial contraction (PAC) & 2 \\
\hline Multifocal PVC & 1 \\
\hline Atrial flutter & 1 \\
\hline Ventricular tachycardia (VT) & 1 \\
\hline Complete AV block & 1 \\
\hline
\end{tabular}

cent years, FMCG has also been used in a wide range of clinical applications in the field of prenatal treatment [1-9]. We herein report our use of MCG for the diagnosis of fetal arrhythmias, and to perform an analysis of fetal autonomic nervous system activity.

\section{MATERIALS AND METHODS}

\subsection{Subjects}

\subsubsection{Diagnosis of Fetal Arrhythmias}

We analyzed ten cases of fetal arrhythmia referred to Iwate Medical University Hospital for detailed testing (Table 1).

\subsubsection{Assessment of Fetal Autonomic Nervous System Activity}

Study subjects comprised 35 fetuses who underwent normal pregnancy (28-39 gestational weeks) and 12 fetuses 
Table 2. Descriptive Characteristics of Normal and Growth Restricted Fetuses (FGR)

\begin{tabular}{|c|c|c|c|}
\hline Item & Normal Fetuses $(\mathbf{n}=\mathbf{3 5})$ & FGR Fetuses $(\mathbf{n}=\mathbf{1 2})$ & P Value \\
\hline \hline & (Max-Min) & (Max-Min) & NS \\
\hline Maternal age (years) & $31.7 \pm 6.46$ & $30.9 \pm 5.79$ & $(42-23)$ \\
\hline Gestational age (weeks) & $(44-20)$ & $35.2 \pm 1.80$ & NS \\
\hline
\end{tabular}

Values are represented as the mean $\pm \mathrm{SD}$

NS: not significant.

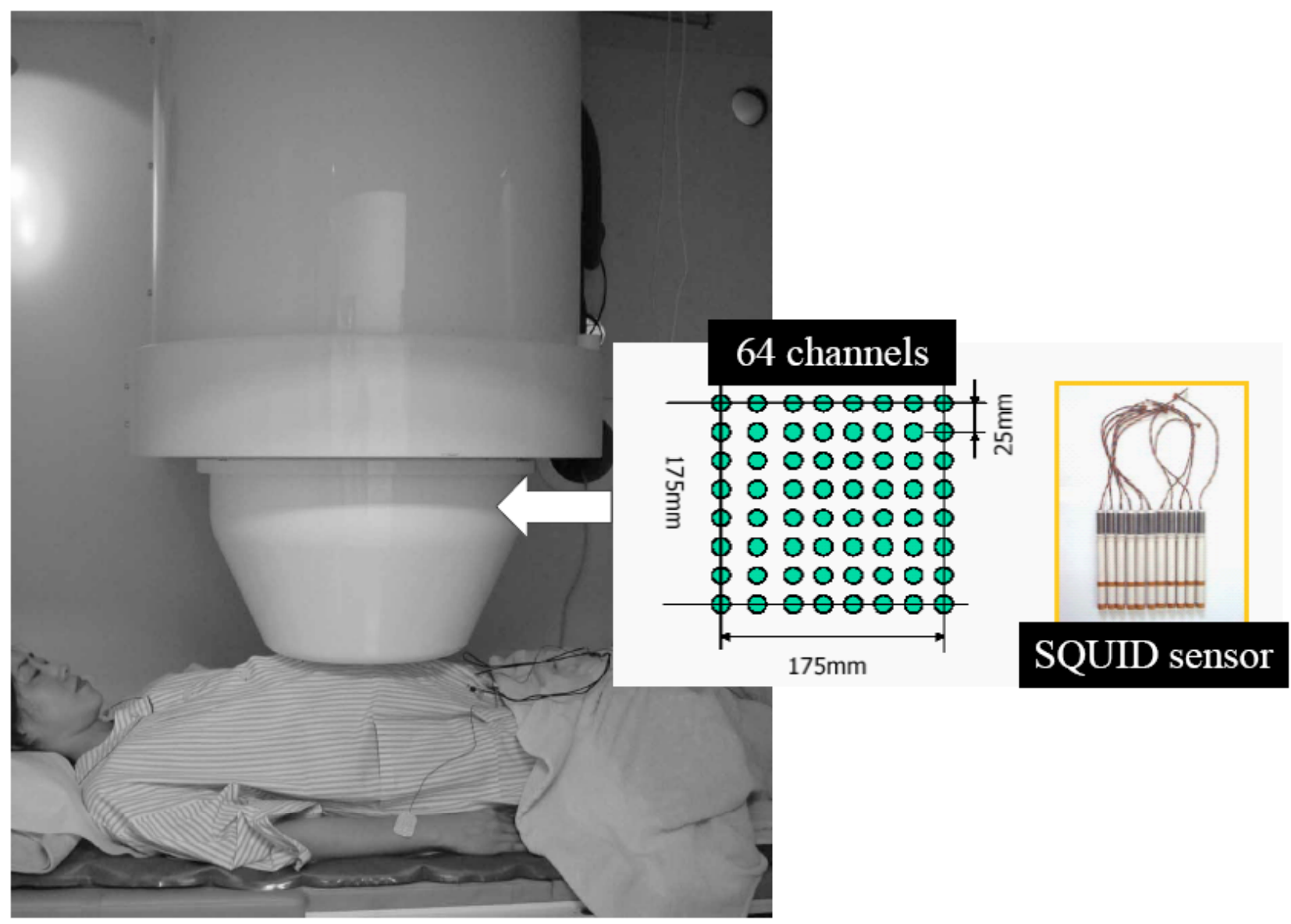

Fig. (1). Monitoring device for magnetocardiography (MCG) incorporating a 64-channel SQUID apparatus in a magnetically shielded room.

with intrauterine fetal growth restriction (FGR) (32-37 gestational weeks) (Table 2). In the normal pregnancy group, fetuses were born at term, without any abnormal neurological signs. In the FGR group, ultrasonography was performed by several specialists for all cases who underwent FMCG. All of these cases presented with asymmetrical FGR with weight $10 \%$ below normal values; none had complications at birth.

Written informed consent was obtained from all subjects after being briefed about the clinical study, which was approved by the Ethics Committee of the School of Medicine, Iwate Medical University (H14-33, H17-2).

\subsection{Equipment}

In order to obtain FMCG measurements, pregnant volunteers assumed a supine position on a bed and were scanned with a 64-channel MCG sensor (Prototype made by the Joint Research Project for Regional Intensive in Iwate Prefecture; SQUID sensor; Hitachi High-Technology Co., Ltd., Tokyo, Japan) installed in a magnetically shielded room (Fig. 1). The position of the fetus was determined by ultrasonography, using the navel and pubic symphysis as reference coordinates (Fig. 2A, 2B). The magnetic field in the $\mathrm{z}$ direction (Bz) adjacent to the body surface was monitored continuously for 5 minutes. A total of two or three measurements were obtained in this manner. As the detected $\mathrm{Bz}$ is a summation of signals from fetal and maternal sources, the maternal QRS waveform was subtracted from the detected $\mathrm{Bz}$ to derive the actual $\mathrm{Bz}$ value. Fetal heart rhythm (approximately 700 beats) was measured for 5 minutes, and the average heart rate was used to derive FMCG data in each case 


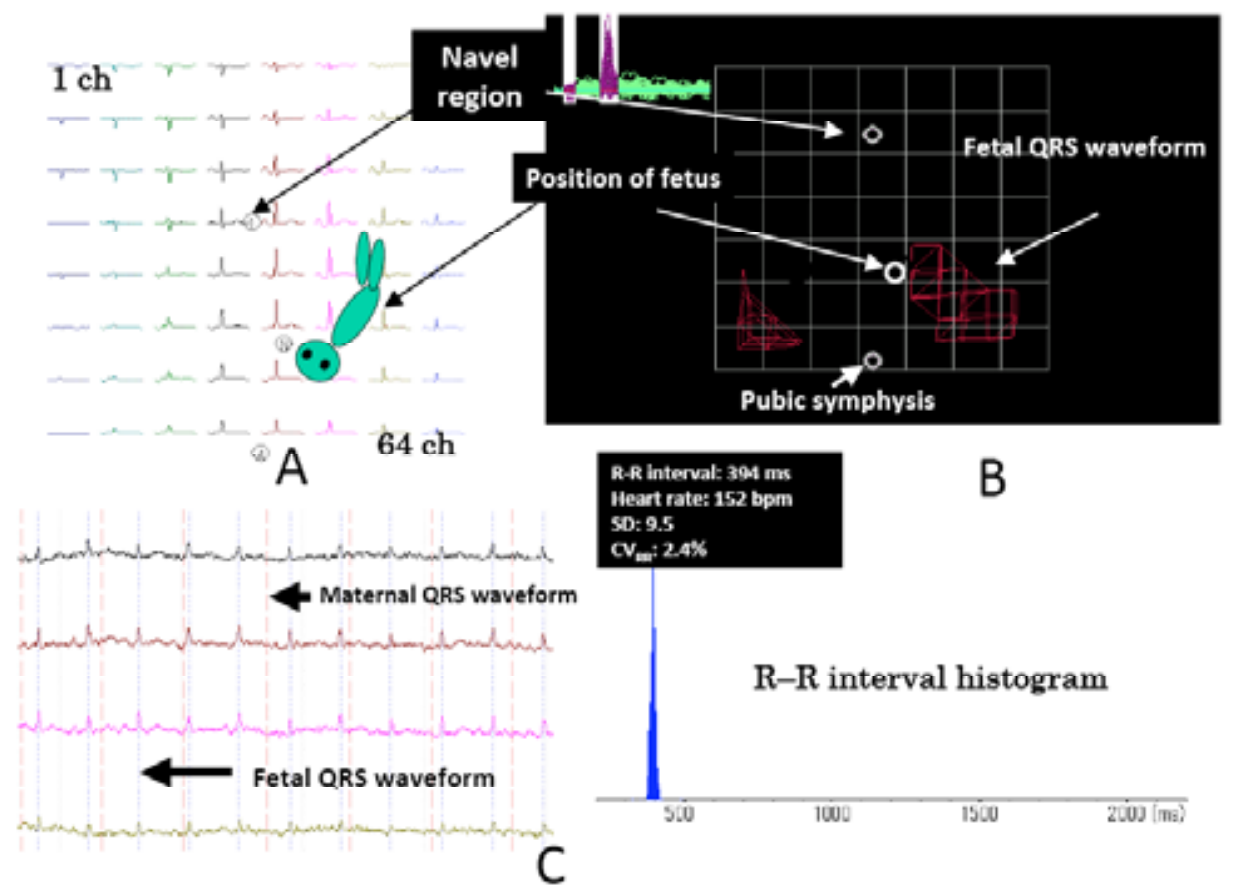

Fig. (2). Method for fetal magnetocardiography (FMCG). Position of the fetus was referenced using standard coordinates based on the navel region and pubic symphysis (A). Detected magnetic field signals, which comprised signals derived from both the mother and fetus, were subtracted from the maternally derived QRS waveform (B). When performing FMCG, a total of 300-500 heartbeats detected per determination were summated to yield mean values $(\mathbf{C})$.

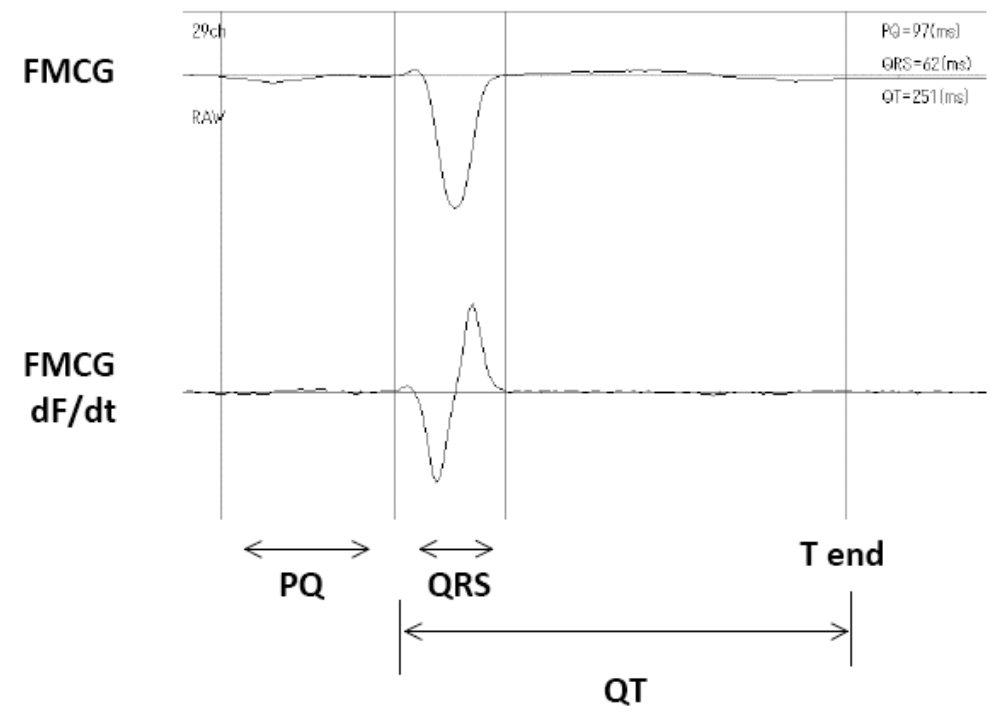

Fig. (3). Determination of PQ, QRS and QT intervals by differentiation. In performing FMCG, a total of $300-500$ heartbeats detected per determination were summated to yield mean values. Initial and termination points of the PQ, QRS and $\mathrm{T}$ wave were defined based on the first derivate of $\mathrm{dF} / \mathrm{dt}$.

(Fig. 2C). The initial and termination values of $P Q, Q R S$ and QT were defined by the maximum points of the first derivate of $\mathrm{dF} / \mathrm{dt}$ (Fig. 3).

$\mathrm{CV}_{\mathrm{RR}}$ was calculated as follows:

Standard deviation,

$S D=\sqrt{\frac{1}{n} \sum_{i=1}^{n}(D i-\bar{M})^{2}}$

and

$$
C V_{R R}=S D / \frac{M}{M} \times 100(\%)
$$

where $\mathrm{n}$ and $D i$ represent the number of $\mathrm{R}-\mathrm{R}$ intervals and the mean of approximately $700 \mathrm{R}-\mathrm{R}$ intervals, respectively, and $M$ represents the total mean of the respective $D i$.

The power spectrum in the frequency domain was derived from frequency-field components using the maximum entropy method of fetal heart rate variability. Based on frequency analysis, the ranges of the LF and HF domains were defined as $0.01-0.15 \mathrm{~Hz}$ and $0.15-0.4 \mathrm{~Hz}$, respectively. 


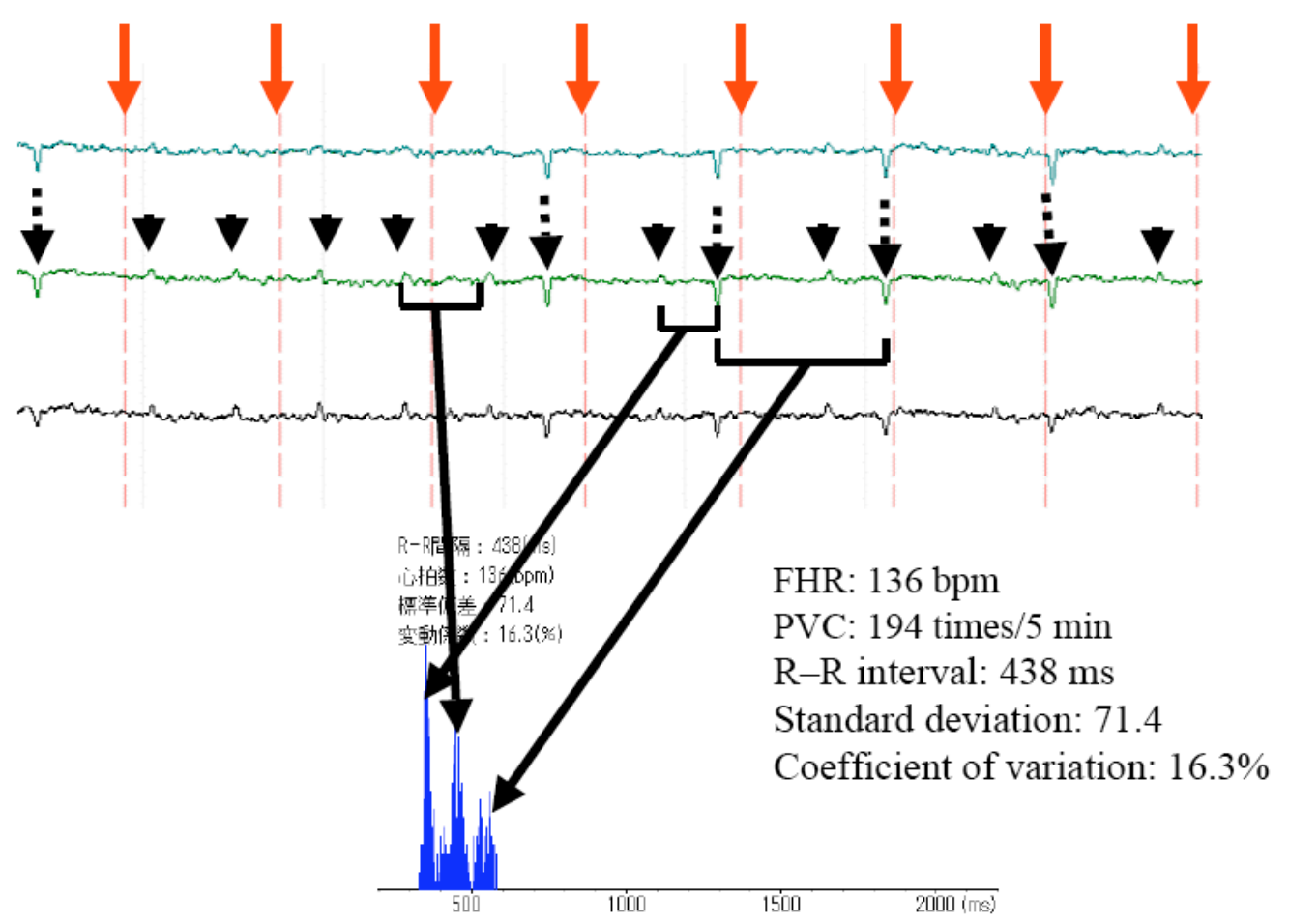

Fig. (4). Representative FMCG recordings in Case 1

Maternal sinus rhythm:

Fetal sinus rhythm:

Premature ventricular contraction (PVC):
FHR: $136 \mathrm{bpm}$

PVC: 194 times/5 min

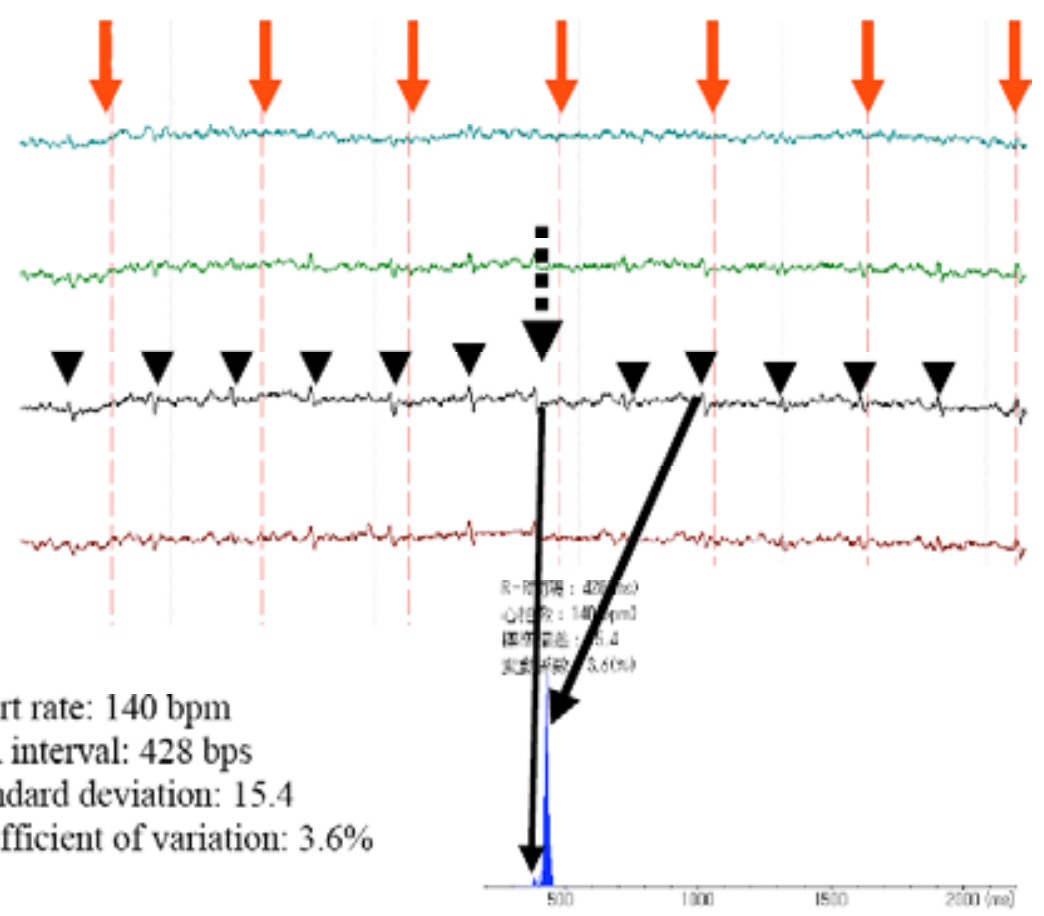

Fig. (5). Representative FMCG recordings in Case 2

Maternal sinus rhythm:

Fetal sinus rhythm:

Heart rate: $140 \mathrm{bpm}$

R-R interval: 428 bps

Standard deviation: 15.4

Coefficient of variation: $3.6 \%$

FHR: $136 \mathrm{bpm}$

PVC: 194 times/5 min

$\mathrm{R}-\mathrm{R}$ interval: $438 \mathrm{~ms}$

Standard deviation: 71.4

Coefficient of variation: $16.3 \%$

Premature atrial contraction (PAC) 


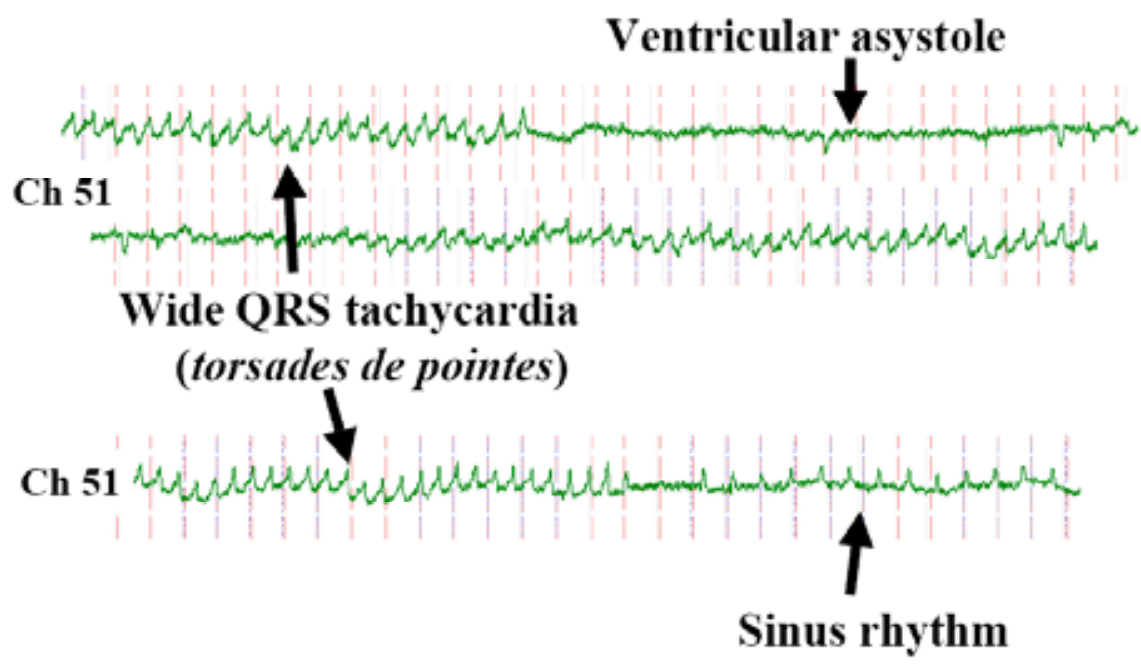

Fig. (6). Representative FMCG recordings in Case 3 Each non-averaged FMCG channel represents wide QRS tachycardia (torsades de pointes), ventricular asystole and sinus rhythm at 28 weeks 3 days gestation.

The respective power values were derived to yield the $\mathrm{LF} / \mathrm{HF}$ ratio. Note that the LF/HF ratio is taken as sympathetic activity of the fetal autonomic nervous system (ANS) $[10,11]$.

\subsection{Statistical Analysis}

Data were analyzed using StatView for Windows Ver. 5.0 (SAS Institute Inc., Cary, NC). The relationships among $\mathrm{CV}_{\mathrm{RR}}, \mathrm{LF} / \mathrm{HF}$ and gestational age in each group were analyzed by linear regression, while inter-group changes in $\mathrm{CV}_{\mathrm{RR}}$ and $\mathrm{LF} / \mathrm{HF}$ over the gestational period in each group were verified by one-way ANOVA. Changes in $\mathrm{CV}_{\mathrm{RR}}$ and $\mathrm{LF} / \mathrm{HF}$ over the gestational period between normal pregnancy and the FGR group were analyzed by two-way ANOVA.

\section{RESULTS}

\subsection{Prenatal Diagnosis of Fetal Arrhythmias}

We describe below three representative cases out of ten, in which FMCG was used to analyze fetal arrhythmias.

Fetal arrhythmia case 1: Premature ventricular contraction (PVC)

The mother was urgently transported to our hospital at 39 weeks 4 days gestation due to fetal bradyarrhythmia. FMCG indicated a prenatal diagnosis of PVC, as shown in Fig. (4). A female infant (birth weight, $3480 \mathrm{~g}$ ) was born by normal vaginal delivery at 41 weeks 0 days gestation. The diagnosis of PVC was confirmed postnatally by ECG. (PAC)

Fetal arrhythmia case 2: Premature atrial contraction

The mother was transported to our hospital at 27 weeks 4 days gestation due to fetal arrhythmia. FMCG indicated a prenatal diagnosis of PAC, as shown in Fig. (5). A male infant (birth weight, $2576 \mathrm{~g}$ ) was born by normal vaginal delivery at 38 weeks 6 days gestation, and the arrhythmia resolved postnatally.

Fetal arrhythmia case 3: Polymorphic ventricular tachycardia
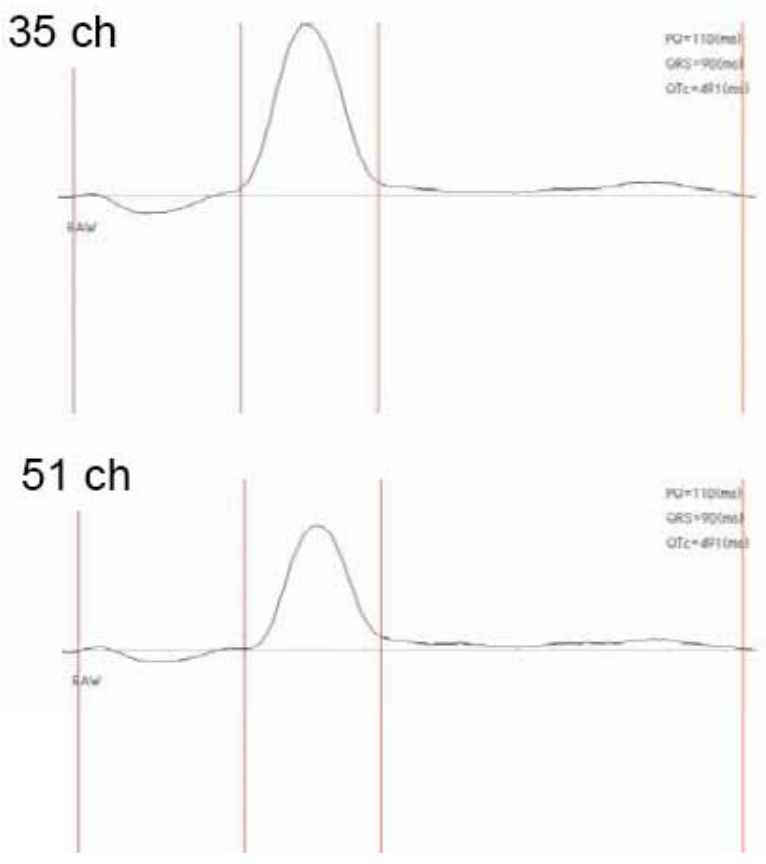

\section{PQ/QRS/QTc 110/90/491 ms}

Fig. (7). Signal-averaged representative MCG recordings in Case 3. Time interval of QRS and QTc (using Bazzet's correction) was determined during signal-averaged sinus rhythm on channel 51 . QRS interval during signal-averaged sinus rhythm at channel 51 was $90 \mathrm{~ms}$, and QTc was $491 \mathrm{~ms}$.

The mother was urgently transported to our hospital at 24 weeks 4 days gestation due to persistent fetal bradycardia and fetal ascites. FMCG findings at 28 weeks 1 day gestation, revealed multiple episodes of non-sustained polymorphic VT and ventricular asystole (Fig. 6). The time intervals for QRS and QTc (using Bazzet's correction) were determined by signal-averaging FMCG during sinus rhythm at an optimal channel setting (channel 51). The QRS interval during a signal-averaged sinus rhythm at channel 51 was $90 \mathrm{~ms}$ and QTc was $491 \mathrm{~ms}$ (Fig. 7). At the instant of diagnosing 


\section{Standard 12-lead ECG after birth}

A

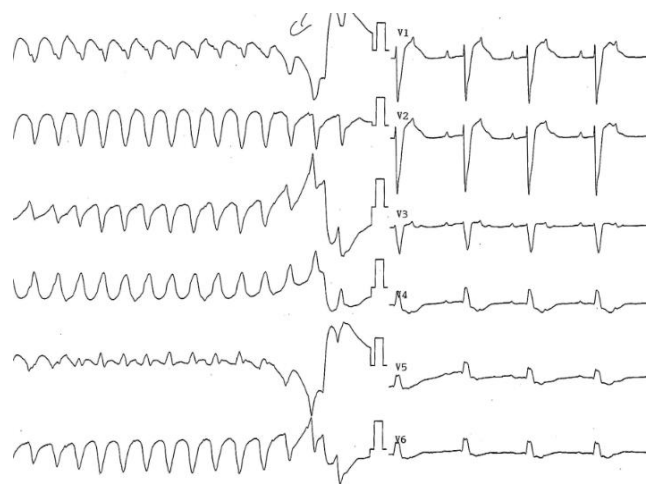

sustained VT - sinus rhythm
B

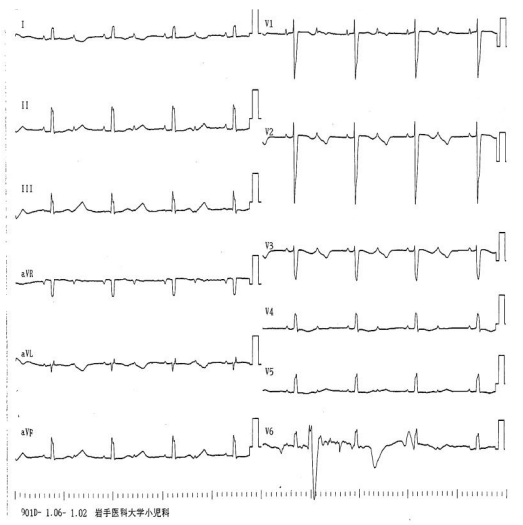

sinus rhythm

Fig. (8). Standard 12-lead ECG immediately after birth of Case 3.

A: Non-sustained polymorphic VT and spontaneous termination followed by 2:1 AV block in precordial leads $\mathrm{V}_{1}-\mathrm{V}_{6}$. B: Sinus rhythm with prolonged QT and 2:1 AV block

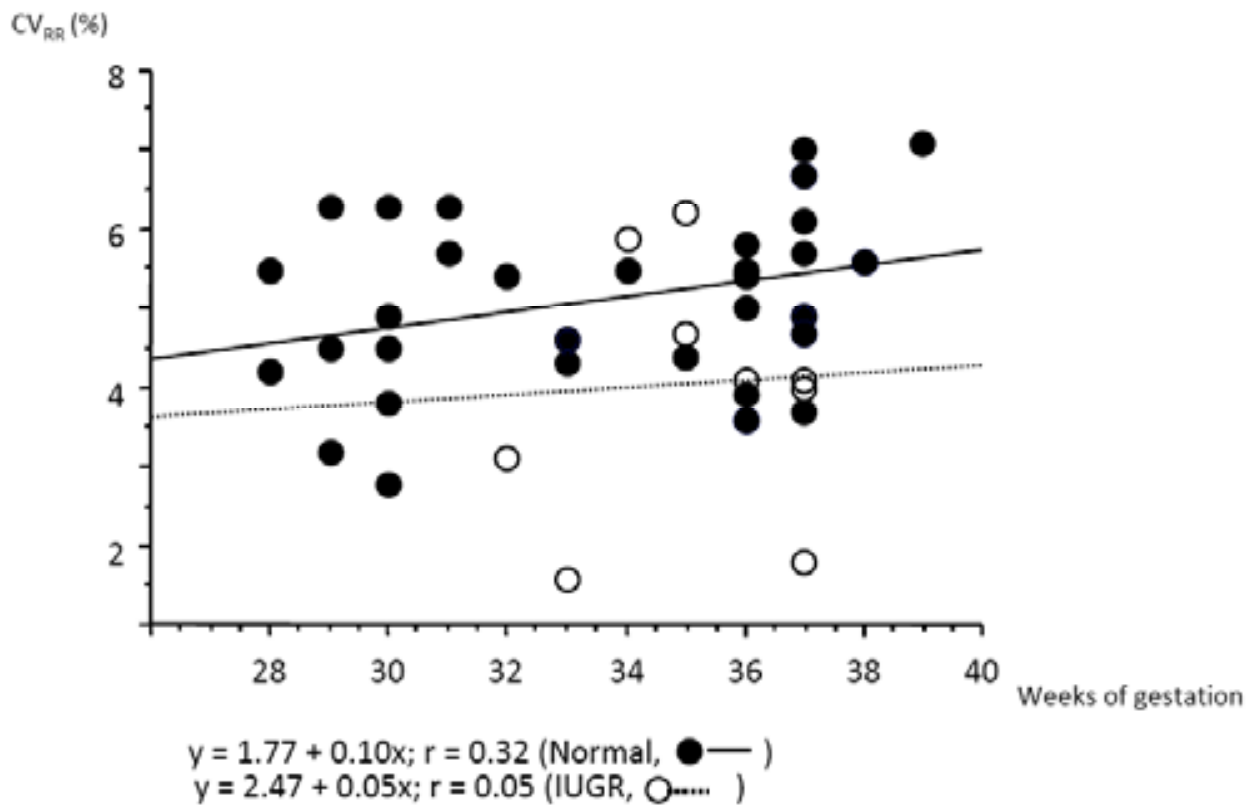

Fig. (9). Correlation between coefficient of variance $\left(\mathrm{CV}_{\mathrm{RR}}\right)$ and gestational age in normal pregnancy and FGR (Normal pregnancy, • ; FGR, ० ).

non-sustained polymorphic $\mathrm{VT}, \mathrm{MgSO}_{4}$ was administered to avoid life-threatening arrhythmia. Administration of a $\beta_{2}$ stimulant (typically employed for the treatment of threatened premature delivery) was avoided. There was no direct evidence for the effectiveness of avoiding QT prolongation using $\mathrm{Mg} \mathrm{SO}_{4}$ by FMCG; this baby was successfully delivered at term. At 37 weeks 1 day gestation, a female infant (birth weight, 2,748 g) was delivered by cesarean section. Standard electrocardiography (ECG) on the day of the birth confirmed non-sustained polymorphic VT, followed by sinus rhythm with 2:1 atrioventricular (AV) block, left bundle branch block (LBBB) and QT prolongation (Fig. 8). Pacemaker treatment was initiated immediately after diagnosis [9].

\subsection{Fetal Autonomic Nervous System Activity (Compari- son of Normal Pregnancy and FGR)}

The normal pregnancy group was divided into three groups for classifying one-way ANOVA of $\mathrm{CV}_{\mathrm{RR}}$ and LF/HF, as follows: Group A, 28-31 weeks gestation $\left(8^{\text {th }}\right.$ month of pregnancy); Group B, 32-35 weeks gestation $\left(9^{\text {th }}\right.$ month of pregnancy); and Group C, 36-40 weeks gestation $\left(10^{\text {th }}\right.$ month of pregnancy).

The value of $C V_{R R}$ in normal pregnancy showed a slightly elevated trend with gestational age $(\mathrm{y}=1.77+$ $0.10 \mathrm{x} ; \mathrm{r}=0.32$ ). In contrast, the value of $\mathrm{CV}_{\mathrm{RR}}$ in the FGR group showed no such trend (Fig. 9). Inter-group changes in 


$$
\mathrm{CV}_{\mathrm{RR}}(\%)
$$

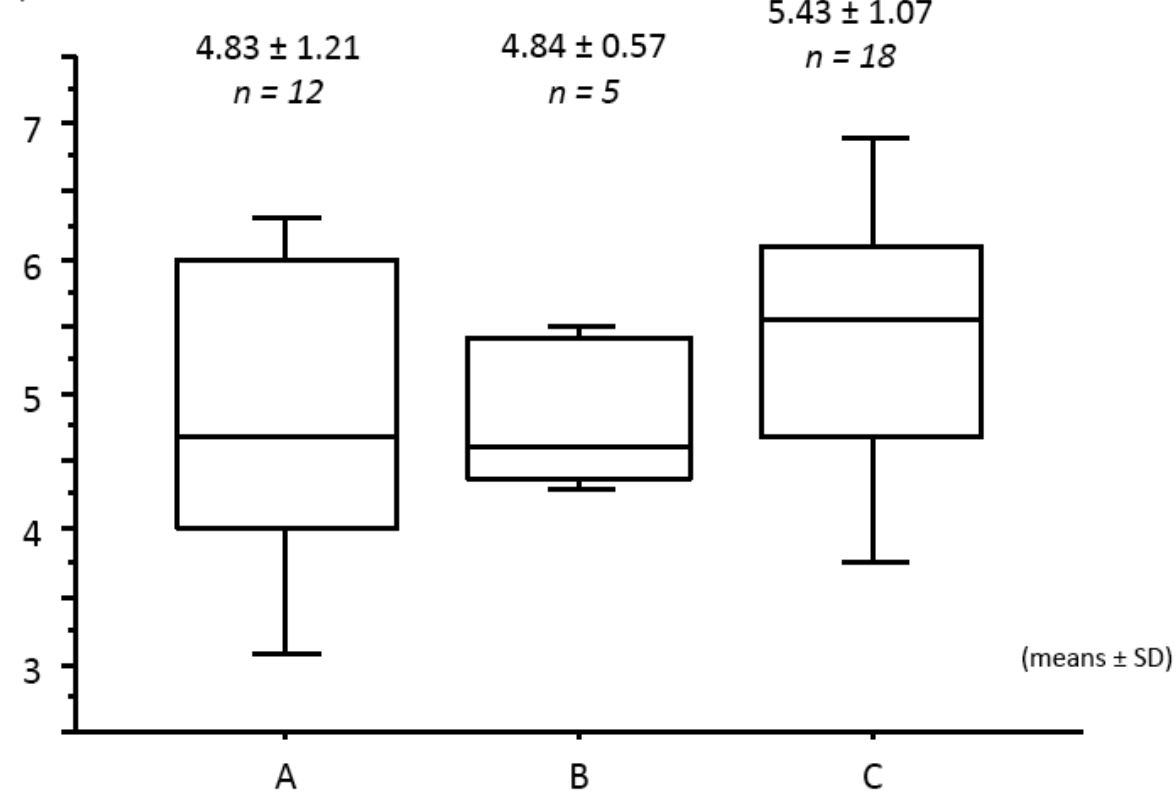

Fig. (10). Inter-group changes in coefficient of variance $\left(\mathrm{CV}_{\mathrm{RR}}\right)$ during normal pregnancy. Group A, 28-31 weeks gestation; Group B, 32-35 weeks gestation; and Group C, 36-40 weeks gestation.

LF/HF ratio

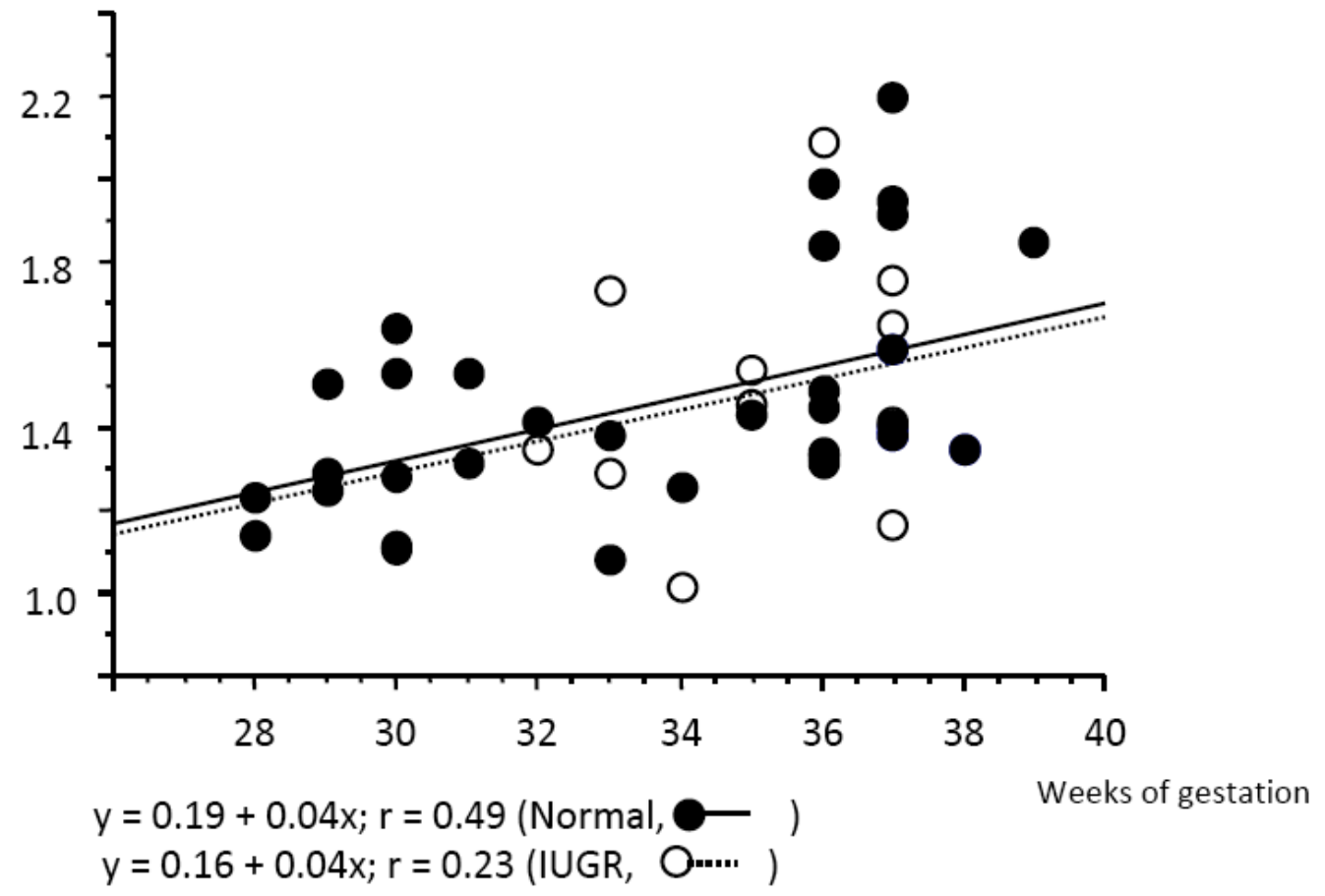

Fig. (11). Correlation between the low frequency/high frequency (LH/HF) ratio and gestational age in normal pregnancy and FGR. (Normal pregnancy, $\bullet$; FGR, ○)

$\mathrm{CV}_{\mathrm{RR}}$ over the gestational age periods in normal pregnancy showed no statistical differences on one-way ANOVA $[7,8]$ (Fig. 10).

The value for LF/HF in both the normal pregnancy group and the FGR group increased with gestational age $(\mathrm{y}=0.19+$ $0.04 \mathrm{x}, \mathrm{r}=0.49$, and $\mathrm{y}=0.16+0.04 \mathrm{x}, \mathrm{r}=0.23$, respectively) (Fig. 11). Inter-group changes in LF/HF in the normal group increased significantly with gestation period (one-way
ANOVA: $\mathrm{P}=0.003$ ) $[7,8]$ (Fig. 12). There were no significant differences in inter-group changes in $\mathrm{LF} / \mathrm{HF}$ ratio between normal pregnancy and FGR.

\section{DISCUSSION}

Recent advances in medical technology and techniques, such as ultrasonography and cardiotocography, have enabled various fetal parameters in the field of perinatal medicine to 


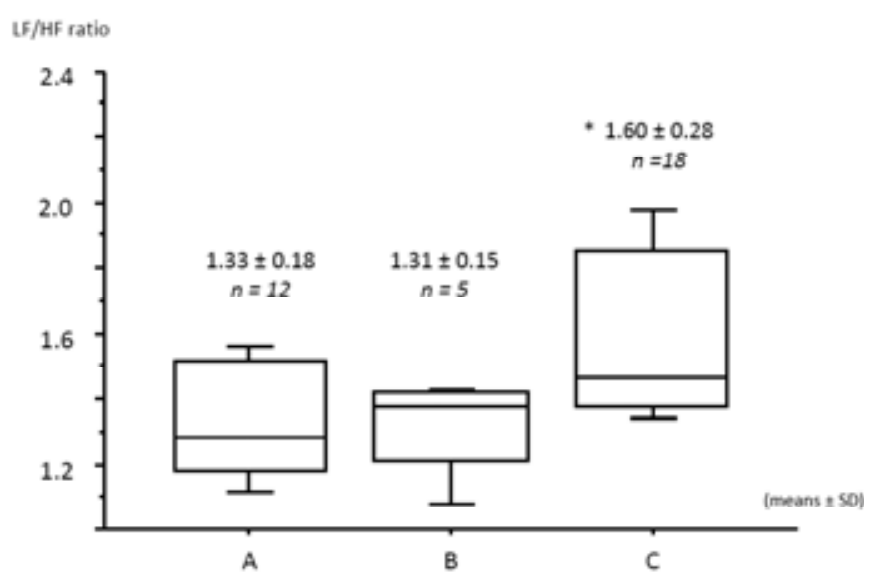

Fig. (12). Inter-group changes in the low frequency/high frequency (LH/HF) ratio during normal pregnancy. Group A, 28-31 weeks gestation; Group B, 32-35 weeks gestation; and Group C, 36-40 weeks gestation.

be obtained in greater detail and with higher precision. For example, current ultrasonography devices can provide detailed evaluations regarding morphology and/or blood flow dynamics. Because this technique diagnoses electrophysiological phenomena based on mechanical information, however, it has many disadvantages from the viewpoint of diagnostic accuracy. In the present study, we developed an FMCG approach by modifying our recently developed 64channel MCG $[12,13]$. The special features of MCG make it possible for the device to perform a 3-dimensional analysis of the magnetic field generated by the electric activities of the heart in both fetuses and adults. FMCG is an extremely safe and non-invasive method for monitoring fetal cardiac activities.

To date, numerous studies have used FMCG to analyze fetal arrhythmias [5, 6, 14-16]. In the cases we have analyzed to date, particularly fetal arrhythmia case 3 , it would have been extremely difficult to reach an accurate diagnosis using conventional testing methods, meaning that it might not have been possible to select appropriate treatment strategies. FMCG has been extremely useful for the accurate diagnosis of fetal arrhythmia and its effective monitoring during pregnancy, enabling these fetuses to be managed appropriately up to 37 weeks gestation. FMCG has also enabled the appropriate planning of birth timing, delivery method and postnatal treatment [9].

In addition to fetal structural anomalies, there are many other aspects of contemporary perinatal medicine in which fetal functional assessment is required, such as intrauterine assessment of fetuses with FGR, and analysis of the cause and timing of onset of cerebral palsy. To assess fetal ANS function, Shields and Schifrin evaluated fetal heart rate variability derived from fetal cardiotocography and respiratory movements [17]. More than $90 \%$ of fetal asphyxia cases were detected by abnormal heartbeat patterns using cardiotocography. Thus, cardiotocography is an indispensable tool that is routinely employed in clinical obstetrics; however, even when abnormal heartbeat patterns are detected, the combined use of ultrasonic Doppler and biophysical profile analysis does not improve the ability to identify undiagnosed cases of asphyxia. These conventional methods are yet to objectively and reliably evaluate functional development of the fetal ANS; they are also unable to consistently achieve prenatal diagnosis of conditions that result from abnormal fetal ANS development, such as cerebral palsy. As a result, unnecessary obstetric interventions are encountered, and the rate of cesarean section continues to increase without any decrease in the incidence of fetal central nervous system impairments, particularly cerebral palsy. This problem highlights the need for novel approaches to prenatal diagnosis $[18,19]$.

MCG can thus be used for analysis of fetal heart rate variability, enabling further improvements to the accuracy of assessments of fetal wellbeing. Although analyses of heart rate variability have been attempted using FMCG, little has been established [20, 21]. To advance the development of this method, we undertook the following studies: (1) elucidation of the developmental stages of fetal ANS over a period of 39 weeks; and (2) comparison of FMCG findings between normal pregnancy and FGR groups. In FGR cases, the difference in fetal size and other obstetric risks are greater, and the correlation decreases when compared with the normal pregnancy group; that is, the heart rate coordination system is non-versatile and highly susceptible to stress [22, 23]. Therefore, it is critical to differentiate on the grounds of perinatal management whether a fetus has low body weight alone, or whether it also has endogenous functional issues.

According to a previous study $[7,8]$, the results of attempted measurements of $\mathrm{CV}_{\mathrm{RR}}$, a value that reflects parasympathetic nervous system (PSN) activity [24, 25], indicated that $\mathrm{CV}_{\mathrm{RR}}$ values exhibited a slightly increasing trend with gestational age in the normal pregnancy group. In addition, we defined the value of the LF/HF ratio as sympathetic nervous system (SNS) activity or the balancing factor between SNS and PNS activities [26]. The LF/HF ratio showed a clear increase with gestational age in the normal pregnancy group [7, 8]; fetal ANS function is known to develop as gestation progresses [26]. These results are consistent with ultrasound analyses of the fetal mouth movement interval and eye-movement phase patterns, in which major changes occur at around gestational weeks 28-33 [23]. This evidence from earlier studies is consistent with the results of the present study. Our results revealed that the maturation process, particularly in SNS activity or the balancing factor between SNS and PNS activities, changes dramatically from 28-39 weeks gestation. For the fetus, therefore, it is very important to avoid preterm delivery, not only in terms of body size and organic maturation, but also for the functional development of ANS.

A previous frequency analysis using FMCG indicated decreased complexity and increased periodicity of the LF and HF ranges in FGR cases [21]. In another previous study, the value of the LF/HF ratio in the IUGR group tended to increase with gestational age; however, the value of $C_{R R}$ did not show a clear trend. This finding may depend on growth restriction or heterogeneity in terms of etiology and severity in FGR cases $[8,27]$. Further investigation of FGR cases, along with detailed analyses of patient background, is therefore required. The development pattern of ANS in the fetal period may thus become an important index for prenatal management in the near future. 


\section{CONCLUSION}

MCG could be used for analysis of both fetal arrhythmia and fetal heart rate variability. MCG thus enables the acquisition of fetal information that could not be obtained by conventional diagnostic techniques, such as ultrasonography and cardiotocography, and represents an extremely useful diagnostic technique.

\section{CONFLICT Of INTEREST}

None declared.

\section{ACKNOWLEDGEMENTS}

This study was supported by the Budding Scientific Research Grant-in-Aid \#17591753 from the Ministry of Education, Science, and Culture of Japan; Joint Research Project for Regional Intensive in Iwate Prefecture; Dreamland Iwate Strategic Research Promotion Project (2005); the Keiryokai Foundation (No. 88) of Iwate Medical University; and the Open Research Translational Research Center Project, Advanced Medical Science Center, Iwate Medical University.

\section{REFERENCES}

[1] Menéndez T, Achenbach S, Beinder E, et al. Prenatal diagnosis of QT prolongation by magnetocardiography. Pacing Clin Electrophysiol 2000; 23(8): 1305-7.

[2] Menéndez T, Achenbach S, Beinder E, et al. Usefulness of magnetocardiography for the investigation of fetal arrhythmias. Am J Cardiol 2001; 88(3): 334-6.

[3] Hosono T, Chiba Y, Shinto M, Kandori A, Tsukada K. A fetal Wolff-Parkinson-White syndrome diagnosed prenatally by magnetocardiography. Fetal Diagn Ther 2001; 16(4): 215-7.

[4] Hosono T, Kawamata K, Chiba Y, Kandori A, Tsukada K. Prenatal diagnosis of long QT syndrome using magnetocardiography: a case report and review of the literature. Prenat Diagn 2002; 22(3): 198200.

[5] Kähler C, Grimm B, Schleussner E, et al. The application of fetal magnetocardiography (FMCG) to investigate fetal arrhythmias and congenital heart defects (CHD). Prenat Diagn 2001; 21(3): 176-82.

[6] Wakai RT, Strasburger JF, Li Z, Deal BJ, Gotteiner NL. Magnetocardiographic rhythm patterns at initiation and termination of fetal supraventricular tachycardia. Circulation 2003; 107(2): 307-12.

[7] Fukushima A, Nakai K, Kanasugi T, Terata M, Sugiyama T. Assessment of fetal autonomic nervous system activity by fetal magnetocardiography. Clin Med Cardiol 2008; 2: 33-9.

[8] Fukushima A, Nakai K, Kanasugi T, Terata M, Sugiyama T. Assessment of fetal autonomic nervous system activity by fetal magnetocardiography: Comparison of normal pregnancy and intrauterine growth restriction. J Pregnancy 2011; 2011: 218162.

[9] Fukushima A, Nakai K, Matsumoto A, Strasburger J, Sugiyama T. Prenatal diagnosis of polymorphic ventricular tachycardia using 64-channel magnetocardiography. Heart Vessels 2010; 25(3): 2703.
[10] Akselrod S, Gordon D, Ubel FA, Shannon DC, Berger AC, Cohen RJ. Power spectrum analysis of heart rate fluctuation, a quantitative probe of beat-to-beat cardiovascular control. Science 1981; 213(4504): 220-2.

[11] Rassi D, Lewis MJ. Power spectral analysis of the fetal magnetocardiogram. Physiol Meas 1995; 16(2): 111-20.

[12] Nakai K, Izumoto H, Kawazoe K, et al. Three-dimensional recovery time dispersion map by 64-channel magnetocardiography may demonstrate the location of a myocardial injury and heterogeneity of repolarization. Int J Cardiovasc Imaging 2005; 22(3-4): 573-80.

[13] Nakai K, Kawazoe K, Izumoto H, et al. Construction of a threedimensional outline of the heart and conduction pathway by means of a 64-channel magnetocardiogram in patients with atrial flutter and fibrillation. Int J Cardiovasc Imaging 2005; 21(5): 555-61.

[14] Simpson LL. Fetal supraventricular tachycardia: diagnosis and management. Semin Perinatol 2000; 24(5): 360-72.

[15] van Leeuwen P, Hailer B, Bader W, Geissler J, Trowitzsch E, Grönemeyer DH. Magnetocardiography in the diagnosis of fetal arrhythmia. Br J Obstet Gynaecol 1999; 106(11): 1200-8.

[16] Wakai RT, Leuthold AC, Cripe L, Martin CB. Assessment of fetal rhythm in complete congenital heart block by magnetocardiography. Pacing Clin Electrophysiol 2000; 23(6): 1047-50.

[17] Shields JR, Schifrin BS. Perinatal antecedents of cerebral palsy. Obstet Gynecol 1988; 71(6): 899-905.

[18] Stanley FJ, Watson L. Trends in perinatal mortality and cerebral palsy in Western Australia, 1967 to 1985. BMJ 1992; 304(6843): 1658-63.

[19] Nelson KB, Dambrosia JM, Ting TY, Grether JK. Uncertain value of electronic fetal monitoring in predicting cerebral palsy. New Engl J Med 1996; 334(10): 613-8.

[20] Anastasiadis PG, Kotini A, Anninos P, Adamopoulos A, Sigalas J, Koutlaki N. Chaotic and periodic analysis of fetal magnetocardiogram recordings in growth restriction. Prenat Diagn 2003; 23(5): 405-9.

[21] Kotini A, Anninos P, Adamopoulos A, Avgidou K, Galazios G, Anastasiadis P. Linear analysis of fetal magnetocardiogram recordings in normal pregnancies at various gestational ages. J Obstet Gynaecol 2001; 21(2): 154-7.

[22] Elbert T, Ray WJ, Kowalik ZJ, Skinner JE, Graf KE, Birbaumer N. Chaos and physiology: deterministic chaos in excitable cell assemblies. Physiol Rev 1994; 74(1): 1-47.

[23] Horimoto N, Koyanagi T, Nagata S, Nakahara H, Nakano H. Concurrence of mouthing movement and rapid eye movement/nonrapid eye movement phases with advance in gestation of the human fetus. Am J Obstet Gynecol 1989; 161(2): 344-51.

[24] Berger RD, Akselrod S, Gordon D, Cohen RJ. An efficient algorithm for spectral analysis of heart rate variability. IEEE Trans Biomed Eng 1986; 33(9): 900-4.

[25] Bigger JT Jr., Steinman RC, Rolnitzky LM, Fleiss JL, Albrecht P, Cohen RJ. Power law behavior of RR-interval variability in healthy middle-aged persons, patients with recent acute myocardial infarction, and patients with heart transplants. Circulation 1996; 93(12): 2142-51.

[26] Kotini A, Anninos P, Adamopoulos A, Avgidou K, Galazios G, Anastasiadis P. Linear analysis of fetal magnetocardiogram recordings in normal pregnancies at various gestational ages. J Obstet Gynaecol 2001; 21(2): 154-7.

[27] Grimm B, Kaehler C, Schleussner E, Schneider U, Haueisen J, Seewald HJ. Influence of intrauterine growth restriction on cardiac time intervals evaluated by fetal magnetocardiography. Early Hum Dev 2003; 74(1): 1-11.

Received: December 31, 2011

(C) Fukushima et al.; Licensee Bentham Open.

This is an open access article licensed under the terms of the Creative Commons Attribution Non-Commercial License (http://creativecommons.org/licenses/by-nc/3.0/) which permits unrestricted, non-commercial use, distribution and reproduction in any medium, provided the work is properly cited. 